\section{¿NORMAL O PATOLÓGICO? EL ENFERMO IMAGINARIO EN TIERRA DE NADIE}

\author{
Alberto Molina Pérez \\ Universidad Paris I Panthéon-Sorbonne \\ albertononi@gmail.com
}

Cómo citar este artículo/Citation: Molina Pérez, A. (2013). "¿Normal o patológico? El enfermo imaginario en tierra de nadie". Arbor, 189 (763): a068. doi: http://dx.doi.org/10.3989/ arbor.2013.763n5005

Recibido: 13 julio 2012. Aceptado: 6 junio 2013.

RESUMEN: ¿Es la frontera entre lo normal y lo patológico una realidad o una ficción? ¿Es la enfermedad solo una cuestión de hecho o también de valores? Presentamos aquí algunos ejemplos de cómo supuestas enfermedades pueden ser inventadas y difundidas con fines de lucro (disease mongering) o por la propia dinámica de la investigación médica. Mostramos cómo la frontera entre la salud y la enfermedad es borrosa y está bajo la influencia de representaciones individuales y sociales, de modos de categorización relativos a cada cultura, y del grado de medicalización de la sociedad. Ahora bien, estas consideraciones no le restan realidad sino que le añaden complejidad, porque las construcciones sociales y las experiencias individuales, por ser subjetivas, no son menos reales. Concluimos que la salud y la enfermedad pertenecen simultáneamente a diferentes tipos de realidad, tanto objetivos como subjetivos, y que lo ficticio puede ser real.

PALABRAS CLAVE: Enfermedad; Realismo; Constructivismo; Ficción; Tráfico de enfermedades; Objetividad; Realidad subjetiva.

\section{NORMAL OR PATHOLOGICAL? THE IMAGINARY INVALID IN NO MAN'S LAND}

Copyright: (C) 2013 CSIC. Este es un artículo de acceso abierto distribuido bajo los términos de la licencia Creative Commons Attribution-Non Commercial (by-nc) Spain 3.0.

ABSTRACT: Is the boundary between the normal and the pathological real or fiction? Are health and disease just a matter of fact or are they value-laden? Here we present some examples of how alleged diseases can be invented and propagated by the industry (disease mongering) or by the methodology of medical science itself. We show that the boundary between health and disease is blurred and depends on individual and social representations, culture relative ways of categorising things and people, and by the society's degree of medicalisation. However, we do not mean that it is not real, rather that it is more complex than expected, as the subjectivity of social constructions and individual experiences makes them no less real. Finally, we conclude that health and disease belong to both objective and subjective kinds of reality, so the fictional can be real.

KEYWORDS: Disease; Realism; Constructivism; Fiction; Disease mongering; Objectivity; Subjective reality. 
Existe en filosofía de la medicina un viejo debate entre naturalistas y normativistas sobre los conceptos de salud y de enfermedad. Los primeros, como Claude Bernard o, más recientemente, Christopher Boorse (1975), dicen que es posible definir la enfermedad sobre una base estrictamente científica, por ejemplo à partir del funcionamiento normal del organismo. Los segundos, como Georges Canguilhem (1966) o H. Tristram Engelhardt (1975) afirman que la noción de enfermedad tiene una dimensión axiológica y que la distinción entre lo normal y lo patológico entraña un juicio de valor ${ }^{1}$. Las dos posturas no son necesariamente incompatibles y algunos autores han propuesto soluciones mixtas (Ereshefsky, 2009; Wakefield, 1992).

En cierta medida, aunque no necesariamente, este debate refleja una discusión epistemológica más amplia entre el objetivismo y el constructivismo (Hoffman, 2001; Murphy, 2009). Los primeros dirían que el concepto de enfermedad descansa sobre hechos empíricos contrastables relativos al cuerpo (o la mente) de un individuo, y que implica por tanto un juicio descriptivo. Los segundos, al contrario, alegarían por ejemplo que la salud y la enfermedad, tanto en general como en sus distintas manifestaciones, son construcciones sociales e implican juicios normativos relativos a un determinado sistema de valores. Por ambas partes, los ejemplos son fáciles de encontrar.

En el siglo XVII, Thomas Sydenham, conocido como el Hipócrates inglés, pensaba que las enfermedades son independientes del observador, que existen en la naturaleza misma, listas para ser descubiertas y clasificadas como clasificamos a las plantas y a los animales. En el siglo XIX, la medicina realizó grandes avances en la comprensión científica de las enfermedades a partir de tres perspectivas o mentalidades diferentes -anatomoclínica, fisiopatológica y etiológica- que comparten una concepción naturalista, es decir, la idea de que la enfermedad es una alteración observable en la estructura y/o funcionamiento normal del organismo y que sus causas son estrictamente naturales. Gracias a esos avances, la medicina moderna es infinitamente superior a la de cualquier época pasada. Son sus propios éxitos los que avalan la postura naturalista-objetivista: en efecto, la medicina empezó a diagnosticar y a curar exitosamente cuando se centró en el estudio científico de la naturaleza, dejando a un lado las creencias irracionales y los juicios morales.

Por otro lado, es difícil negar que en algunos casos la frontera entre lo normal y lo patológico no preexiste y que las normas relativas a la salud y la enfermedad son definidas de acuerdo con nuestros intereses. Mi- chel Foucault (1961) diría que esa clasificación es una herramienta de control social. En efecto, los "locos", antes de ser considerados como enfermos e internados en manicomios, compartieron la misma suerte que los leprosos, los enfermos venéreos, los "depravados", los pobres y los delincuentes. Todo eso formaba una categoría un tanto difusa que mezclaba la enfermedad con el pecado y la exclusión social. Más adelante, en el siglo XIX, en Estados-Unidos, los esclavos negros que tenían ansias de libertad eran considerados como enfermos mentales, afectados por un trastorno llamado "drapetomanía". Del mismo modo, hasta los años ochenta, en la Unión Soviética, los disidentes políticos eran declarados enfermos mentales e internados en hospitales de alta seguridad. Y hasta 1992, para la Organización Mundial de la Salud, los homosexuales eran clasificados como enfermos mentales ${ }^{2}$.

De entre los muchos aspectos y asuntos abordados en el debate, la cuestión que nos preocupa aquí especialmente es saber si existe realmente una frontera entre lo patológico y lo no-patológico; o, dicho de otro modo, si esa frontera es una realidad o una ficción. No se trata de encontrar una definición general para los conceptos de enfermedad y de salud. No nos interesa saber si todas las enfermedades tienen algo en común ni nos preocupa que sea un género natural. Obviaremos el problema de su explicación y admitiremos sin reparos su carácter multidimensional (biológico, psicológico, social, religioso, etc.). En este sentido, nos parece oportuna la distinción anglosajona entre disease, illness y sickness que remiten respectivamente a los aspectos biomédico, personal y social. Lo que sí queremos averiguar en este artículo es de qué madera está hecha la frontera que separa una persona enferma de una que no lo está, y si la respuesta a esa pregunta depende del tipo de enfermedad.

De acuerdo con lan Hacking (2001), hablar de la construcción social de algo significa que ese algo se da por supuesto y aparece como inevitable cuando en realidad no lo es, sino que podría ser de otro modo y no está determinado por la naturaleza misma de las cosas. A menudo se usa para decir que algo no es real, que no existe "de verdad". Decimos por ejemplo que las razas humanas son construcciones sociales porque esa clasificación de la humanidad no tiene fundamento biológico. Aplicado a la distinción entre salud y enfermedad, el constructivismo social podría llevarnos a la misma conclusión. Sin embargo, una construcción social también puede ser real en otro sentido. El dinero, la pobreza, el fútbol, el idioma español, el Estado y las fronteras entre Estados... son cosas que sí existen 
precisamente porque han sido construidas y son mantenidas por la sociedad.

Hoy en día, se escuchan cada vez más voces que acusan a las empresas farmacéuticas de inventar y promocionar enfermedades a escondidas de la sociedad $^{3}$. Según ellos, muchas enfermedades serían ficciones creadas por la industria para vender medicamentos a gente que no los necesita. En la primera parte del artículo, veremos que algunas enfermedades son ejemplos claros de ficciones patrocinadas por la industria. Pero, como trataremos de matizar en la segunda parte, la creación y manipulación de enfermedades obedece a mecanismos más complejos, y no sería posible sin la participación de los profesionales de salud, de los enfermos y de la sociedad en general. Esas consideraciones llevarán a situar la frontera entre lo normal y lo patológico en esa tierra de nadie que separa la realidad de la ficción.

\section{TRÁFICO DE ENFERMEDADES}

La noción de disease mongering puede traducirse como tráfico o promoción de enfermedades e incluye tanto su invención como su comercio. En los últimos años han ido acumulándose numerosos hechos y argumentos, algunos ya bastante conocidos, que apuntan hacia la responsabilidad de la industria farmacéutica. Cabe señalar que los industriales no son los únicos actores responsables de esta situación, pero los hechos que se les reprochan están bien documentados y son muy llamativos, por lo que permiten entender claramente el proceso de fabricación de enfermedades y enfermos.

Hace unos treinta años, Henry Gadsden, director de Merck (una de las mayores empresas farmacéuticas mundiales), confió a la revista Fortune su desesperación al ver que el mercado potencial de su empresa estaba confinado solo a los enfermos. Declaró que soñaba con producir medicamentos destinados... a los sanos, para poderlos "vender a todo el mundo" como si fueran chicles (Cassels \& Moynihan, 2005). Tres décadas más tarde, el sueño de Henry Gadsden se hizo realidad. Un documento publicado en 2003 , destinado a los dirigentes de la industria farmacéutica, indica que la capacidad de esa industria para crear mercados de enfermedades nuevas se traduce en ventas que alcanzan billones de dólares, y que los años venideros serán testigos privilegiados de la creación de enfermedades patrocinadas por la industria (Coe, 2003).

Entre los años 2000 y 2003, casi todas las grandes compañías farmacéuticas pasaron por los tribunales de EEUU, acusadas de prácticas fraudulentas; ocho fueron condenadas a pagar más de 2,2 billones de dólares de multa y cuatro de ellas reconocieron su responsabilidad por actuaciones criminales que han puesto en peligro la salud y la vida de miles de personas (Angell, 2005, pp. 217-236).

En 2004, el Real Colegio de Médicos de familia británico acusó a la industria farmacéutica de poner en peligro los sistemas públicos de salud al inventar enfermedades y fomentar la prescripción indebida de medicamentos costosos para incrementar sus ventas. Según la Dra. Maureen Baker, secretaria honoraria del colegio, "siempre es difícil trazar una línea sencilla entre lo normal y lo patológico, pero la industria tiene interés en trazar una línea que incluya la mayor parte posible de la población en la categoría de los enfermos. Después de todo, cuanto mayor sea ese grupo más medicamentos podrán vender." (Royal College of General Practitioners, 2004). El Colegio cita las formas leves de hipertensión, colesterol alto, osteoporosis, ansiedad y depresión como ejemplos de condiciones donde la industria trata de mover las líneas para vender medicación a gente que no la necesita.

En 2005, un comité del Parlamento inglés publicó un informe sobre la influencia de la industria farmacéutica donde denuncia los efectos perversos de una mercadotecnia que antepone los resultados económicos a la salud de las personas (House of Commons Health Committee, 2005). Según este comité, la industria ejerce una influencia excesiva sobre los médicos, los investigadores, las agencias reguladoras, las asociaciones de enfermos, los medios de comunicación y los representantes políticos. Las consecuencias principales de esta situación son, por un lado, los riesgos para la salud que entraña un consumo excesivo de fármacos ${ }^{4} y$, por otro lado, la creciente medicalización de la sociedad.

Los riesgos que entraña el consumo de fármacos son bastante conocidos por la multiplicación reciente de los escándalos. Uno de ellos es el Vioxx ${ }^{\circledR}$, un antiartritis de la compañía Merck antes citada. Se estima que provocó hasta 140.000 infartos y decenas de miles de muertos en EEUU, a los cuales hay que sumar los de otros países. Este medicamento, que se vendió por miles de millones de dólares anuales, fue recetado indiscriminadamente y a gran escala por los médicos, después de una campaña de promoción muy intensa. Los estudios que mostraban la toxicidad del fármaco fueron ocultados por la compañía.

En cuanto a la creciente medicalización de la sociedad, es decir, según el comité, la creencia de que cada 
problema puede ser resuelto con fármacos: "una pastilla para cada problema", el informe del parlamento inglés señala que esa tendencia no ha sido creada por la industria farmacéutica, pero sí incentivada por ella. La industria, dice el informe, actúa como "traficante de enfermedades" (disease-monger), con el propósito de clasificar un número creciente de individuos como "anormales" que deben ser tratados con fármacos. Cuanta más gente se vea a sí misma como aquejada de algún problema médico, mayor será el mercado y mayores los beneficios económicos. Esto es especialmente claro, dice el informe, en el caso de los antidepresivos. Solamente el 5\% de los antidepresivos son recetados para casos de depresión severa, y dos de cada tres son recetados a personas que simplemente se sienten tristes o que sufren por situaciones y circunstancias difíciles. Pero la tristeza, recuerda el informe del comité, forma parte del abanico de las emociones humanas, no es una enfermedad.

En 2006 fue organizado en Australia el primer congreso internacional sobre disease mongering cuyas actas fueron publicadas en un número temático de la revista PloS medicine. El artículo introductorio (Moynihan \& Henry, 2006) menciona tres formas principales de "tráfico": (1) medicalizar aspectos de la vida cotidiana (como la menopausia); (2) presentar problemas leves como si fueran enfermedades serias (como el síndrome del colon irritable); (3) identificar factores de riesgo con enfermedades (como el colesterol alto y la osteoporosis). La primera de esas estrategias es quizás la más rentable ya que permite salirse del ámbito restringido de la terapia, teóricamente limitado a los enfermos, para abordar el ámbito de la optimización, abierto al conjunto de la población.

El ejemplo más conocido es el Viagra ${ }^{\circledR}$. Pfizer consiguió que lo que debía haber sido un tratamiento para hombres con disfunción eréctil causada por la diabetes o por una intervención quirúrgica se convirtiera en un producto consumido por el conjunto de la población masculina (Lexchin, 2006). Pfizer afirmaba que más de la mitad de la población masculina mayor de 40 años tenía dificultades para alcanzar o mantener una erección y que el Viagra ${ }^{\circledR}$ podía ayudarles, independientemente de las causas e independientemente de la frecuencia del problema. Es decir, que frente a un fallo ocasional causado por los nervios, el cansancio, el estrés laboral o las preocupaciones familiares, la mejor o la única respuesta según Pfizer era la medicación. Ahora bien, los usuarios de Viagra ${ }^{\circledR}$ no se limitan a los mayores de 40 años ni a aquellos que sufren problemas de erección. Entre 1998 y 2002, el merca- do de mayor crecimiento era el de los hombres con edades comprendidas entre los 18 y los 45 años (Delate, Simmons, \& Motheral, 2004) que no querían tratar una disfunción sino mejorar lo "normal".

Rápidamente, los industriales se movilizaron para que las mujeres pudieran disfrutar de un "tratamiento" similar, pero les faltaba la enfermedad correspondiente. Un año antes de que Viagra ${ }^{\circledR}$ saliera al mercado, la industria farmacéutica liderada por Pfizer financió una serie de congresos para definir una enfermedad nueva: la disfunción sexual femenina (Forcades i Vila, 2006; Moynihan, 2003). En 1997, 9 compañías farmacéuticas organizaron y financiaron un encuentro cuyo tema era la ausencia de una definición para ese trastorno. La mitad de los asistentes eran representantes de farmacéuticas, y solamente fueron invitados aquellos investigadores que tenían experiencia o interés en colaborar con la industria. Dieciocho meses más tarde, en Boston, tuvo lugar la primera conferencia internacional para la elaboración de un consenso clínico sobre la disfunción sexual femenina. La conferencia fue financiada por ocho compañías farmacéuticas y 18 de los 19 autores de la nueva definición tenían relaciones económicas o de otro tipo con un total de 22 farmacéuticas. Un año más tarde, en 1999, otra conferencia organizada en Boston era financiada por 16 farmacéuticas; la mitad de los participantes tenía vínculos con la industria. Ese mismo año, un estudio afirmaba que el $43 \%$ de la población femenina de EEUU padecía el trastorno nuevamente definido (Laumann, Paik \& Rosen, 1999). Dos de los tres autores de ese estudio tenían vínculos con la industria. En los encuentros de 2000 y 2001, ya eran 22 las compañías patrocinadoras, encabezadas por Pfizer.

Para identificar la "población enferma" se utilizó un cuestionario con una lista de siete síntomas o problemas críticos. Cada uno de ellos era suficiente para diagnosticar la disfunción sexual si una mujer lo había experimentado durante dos meses o más en el último año. Es decir que las mujeres que no tuvieran deseo sexual durante al menos dos meses quedarían automáticamente etiquetadas de "disfuncionales", independientemente de si estaban de luto por la muerte de su pareja, agobiadas por el trabajo, atrapadas en una relación insatisfactoria o gozando de una etapa de plenitud interior. En cuanto a los otros "síntomas", relacionados con la insatisfacción sexual, tampoco se contempló que el problema pudiera ser debido a su pareja.

En 2004, la comercialización del primer medicamento contra la disfunción sexual femenina, un par- 
che de testosterona de la compañía Procter \& Gamble, fue rechazada por la agencia del medicamento de EEUU (Moynihan, 2003). Incluso antes de que fuera evaluado, Procter \& Gamble ya había gastado cien millones de dólares en una campaña de promoción que debía aparecer como de sensibilización y de educación sanitaria antes que como propaganda para un producto pendiente de comercialización. Su estrategia estaba enfocada hacia los profesionales de la salud, los medios de comunicación y el público en general, con el objetivo de moldear su percepción de los problemas sexuales femeninos y del modo de tratarlos. Según la psiquiatra Leonore Tiefer (2006), el producto que realmente estaba vendiendo la compañía en esa etapa no era el medicamento sino la enfermedad.

\section{REPRESENTAR, CATEGORIZAR, MEDICALIZAR}

Las técnicas utilizadas por la industria farmacéutica para fabricar enfermedades se pueden ordenar en tres ejes:

1) Representación: cambiar la representación que la gente tiene de sí-misma y de los demás, en su cuerpo y su comportamiento; alterar la percepción de lo normal y lo anormal, lo aceptable y lo inaceptable; promover el auto-diagnóstico.

La representación implica el conjunto de la sociedad: los profesionales de la salud, los pacientes, y todos los demás.

2) Categorización: crear definiciones nuevas, modificar las antiguas, y desplazar la frontera entre lo normal y lo patológico.

La categorización es competencia exclusiva de la medicina, pero está bajo la influencia de las representaciones sociales.

3) Medicalización "Una pastilla para cada problema": fomentar la resolución farmacológica de los problemas; alterar la relación terapéutica promoviendo la auto-medicación y la prescripción sistemática (convertir los médicos en proveedores de fármacos).

Antes de seguir adelante es conveniente matizar varios puntos. En primer lugar, muchas de esas técnicas tienen una cara positiva que nos permite disfrutar de un estado general de salud bastante bueno. Financiar campañas de sensibilización y de reconocimiento, o fomentar la educación sanitaria y el autodiagnóstico, no son actividades reprobables en sí. Al contrario, contribuyen a la detección precoz de enfermedades como el cáncer de mama o el cáncer de la próstata.
Por otro lado, la formación destinada a los médicos es muy importante para la actualización de sus conocimientos, tanto a nivel diagnóstico como terapéutico. La medicalización de los factores de riesgo puede tener un interés preventivo. Y el "tratamiento" de los pequeños problemas cotidianos responde a una demanda de la sociedad. En segundo lugar, la disminución de los umbrales de tolerancia es una consecuencia de la elevación de los niveles de vida y de salud de la población. Según Amartya Sen (2002), la percepción de la enfermedad está en relación directa con el nivel de salud y de educación de la población: en EEUU y en las regiones ricas de la India, la gente tiene una mayor percepción de la enfermedad que en las regiones más pobres de la India. Según el premio Nobel, parece incluso haber una fuerte correlación con la esperanza de vida: la gente se siente más enferma cuando su esperanza de vida es más elevada. Cuando hay menos problemas graves de los que preocuparse, se presta mayor atención a los problemas pequeños, y la tolerancia al dolor y al sufrimiento disminuye. Una continuación lógica de ese movimiento es la voluntad de optimización. Todo eso no sería posible si no fuera por el éxito extraordinario de las farmacéuticas en inventar moléculas realmente eficaces. En tercer lugar, si bien es cierto que la industria tiene interés en incentivar ese fenómeno social, echarle toda la culpa encima solo sirve para disimular la responsabilidad de los demás actores. La industria produce fármacos, pero los médicos los recetan, y la sociedad los exige.

lan Hacking (2004) cuenta que en 2002 se constató una "epidemia" de autismo en la Silicon Valley de California. Un médico, dice, propuso una explicación convincente del fenómeno: los niños afectados son casi todos hijos únicos de padres treintañeros o más que se mueven en un ambiente compuesto mayoritariamente por jóvenes, técnicos o ingenieros, que no tienen hijos. Al no tener contacto ni familiaridad con el mundo de los niños, esta comunidad carece de normas, le faltan referencias. Por consiguiente, cuando el comportamiento de un niño resulta molesto para los padres, creen que no es normal y lo llevan al psiquiatra. En otro contexto, los padres dirían que el niño es pesado o difícil, pero no irían a consultar. Vemos aquí, según Hacking, cómo la representación subjetiva de lo normal puede interferir con un diagnóstico médico supuestamente objetivo.

Este ejemplo es especialmente interesante porque a pesar de que la categorización del autismo ha variado con el tiempo, alternando entre una y varias ${ }^{5}$, podrían existir factores biológicos detrás de algunas 
de sus manifestaciones. Si hay un marcador biológico, sea genético o neurológico, entonces parece haber un hecho empírico independiente que permite confrontar el diagnóstico, e incluso la categoría misma de autismo con otra cosa que la opinión de los expertos. Podría entonces darse el caso que algunos de los que fueron en su día clasificados como enfermos dejen de serlo de acuerdo con los nuevos criterios. Para un naturalista, el ejemplo del autismo muestra claramente que existe una diferencia entre la realidad -en este caso la distinción entre lo normal y lo patológicoy nuestras normas y representaciones de la misma. Pero ¿qué pasa cuando la categorización y el diagnóstico de una enfermedad no pueden apoyarse en una base biológica? Es lo que ocurre con muchos trastornos mentales, como la depresión.

Philippe Pignarre (2001) explica con bastante detalle el proceso de categorización de ese trastorno y cómo ese proceso es en gran parte responsable de su crecimiento exponencial en los países desarrollados. El autor cita tres explicaciones clásicas para ese fenómeno. La primera es social: la multiplicación de los casos de depresión sería un reflejo y una consecuencia de la evolución de la sociedad, cada vez más inhumana, cruel y estresante. La segunda explicación es médica: los depresivos tendrían una predisposición orgánica que se diagnostica cada vez mejor. La tercera explicación es analítica: los depresivos tendrían una predisposición psicológica, por culpa de un trauma infantil, lo cual supone que los problemas familiares también deberían estar en aumento. A esas tres explicaciones se puede añadir una cuarta, que ya conocemos, según la cual la industria farmacéutica manipula la opinión para vender sus antidepresivos. Philippe Pignarre propone otra explicación que apunta hacia la psiquiatría moderna, la metodología de investigación farmacológica, y los propios pacientes.

El Manual Diagnóstico y Estadístico de los trastornos mentales (DSM), que recoge el consenso alcanzado por diferentes grupos de psiquiatras norteamericanos, es una herramienta que sirve para que en un mismo individuo reciba el mismo diagnóstico ${ }^{6}$. Ese manual define la depresión por sus síntomas, no por sus causas, porque no hay consenso sobre las causas. Por consiguiente, según Pignarre, el DSM no nos dice de qué sufren realmente los pacientes (las causas de su trastorno), sino cuáles son los criterios que permiten clasificar a una persona en la categoría "depresivo". Esa confusión sobre el alcance de las herramientas de diagnóstico es la primera condición de la epidemia.
Otras dos condiciones son la ausencia de un marcador biológico fiable, por un lado, y la existencia de medicamentos eficaces, por el otro. Esto es muy importante, explica Pignarre, porque la ausencia de un marcador biológico fiable permite que sean los propios medicamentos los que definan la enfermedad. En efecto, según otros autores, la epidemia de depresión podría ser una consecuencia del descubrimiento de los antidepresivos. El motivo es el siguiente. Cuando los investigadores desarrollan una nueva molécula y la prueban sobre grupos de pacientes para ver cuáles son sus efectos, todos los sujetos del ensayo clínico no reaccionan igual. Entonces los investigadores pueden y deben hacer variar las características del grupo de pacientes, es decir, los criterios de inclusión, hasta que el grupo "responda" satisfactoriamente a la molécula candidata. De este modo, a medida que cambian los criterios de inclusión de los pacientes, se va dibujando el conjunto de síntomas sobre los cuales la molécula es eficaz. Eso significa que el trastorno que padecen los pacientes de ese grupo viene a ser definido como aquello sobre lo que actúa el antidepresivo. Por consiguiente, cuando se descubre una nueva familia de fármacos, aparece al mismo tiempo una nueva categoría de depresión.

Pero eso no es todo. El número de sujetos que participan en los ensayos clínicos aumenta progresivamente entre las fases I, II y III de la experimentación hasta alcanzar miles e incluso decenas de miles de personas. En efecto, son las estadísticas las que aportan la prueba de la eficacia de un fármaco. Ahora bien, los ensayos de fase III no tienen lugar en un laboratorio aislado de la sociedad sino en la vida real. Es decir, que mientras recogen información farmacológica los ensayos clínicos instalan la enfermedad, definida por los criterios de inclusión, en la vida cotidiana de miles o decenas de miles de personas. Después, cuando el tratamiento ya ha sido aprobado, hay todavía estudios de farmacovigilancia (fase IV) que implican esta vez a cientos de miles de personas. Paradójicamente, parece ser el propio proceso de investigación el que contribuye a difundir la enfermedad a medida que descubre los fármacos para tratarla.

El caso de la depresión muestra que la ausencia de un marcador biológico fiable no permite distinguir entre descubrimiento e invención, o entre realidad nosológica y ficción científica. ¿A qué corresponden pues las categorías psiquiátricas: a diferentes tipos de patología, o a diferentes clases de fármacos? En otras palabras, cuando se clasifica a una persona como depresiva, ¿qué es lo que se está diciendo: que esa per- 
sona tiene un trastorno mental, o que esa persona se sentirá mejor tomando antidepresivos? Desde luego no es lo mismo. En un caso la persona está enferma y en el otro no. Sin embargo, el sufrimiento es idéntico, y los efectos del tratamiento también.

Sufrir una pulmonía y estar enfermo no es exactamente lo mismo. La diferencia tiene mucho que ver con el conjunto de prácticas y de representaciones sociales con los que va asociada la etiqueta "enfermo". El hecho de estar enfermo, es decir, el hecho de ser clasificado como tal, puede aportar grandes ventajas y graves inconvenientes. Además de la simpatía y de la conmiseración de la gente, la enfermedad le puede evitar a uno ser condenado en los tribunales, ser enrolado en el ejército, o tener que ir a trabajar. Al darse de baja por enfermedad, uno se sigue beneficiando de un salario fijo, puede recibir compensaciones del seguro, y tiene acceso a ayudas y servicios denegados a los sanos. Desde un punto de vista individual, uno puede encontrar en la enfermedad una explicación para su sufrimiento. $Y$ las actitudes tanto personales como sociales frente a un determinado problema tampoco serán las mismas según que ese problema se considera o no como enfermedad. No es lo mismo ser un vago que tener el síndrome de "déficit motivacional". No es lo mismo ser un borracho que tener "dependencia patológica al alcohol con predisposiciones biológicas". El lado negativo de ser un enfermo, es que uno puede sufrir rechazo, exclusión social, discriminaciones de todo tipo, e incluso privación de libertad.

Para las farmacéuticas, la medicina y el sistema de salud en general también hay ventajas e inconvenientes de categorizar un problema como patológico. Cada enfermedad nueva supone una nueva carga para los sistemas de sanidad que garantizan el acceso a los tratamientos. Al contrario, para la medicina y las farmacéuticas, eso puede resultar muy lucrativo. Si una molécula es autorizada como tratamiento para alguna enfermedad, como la disfunción sexual femenina, entonces las farmacéuticas reciben cuantiosas subvenciones indirectas del Estado cada vez que este reembolsa el fármaco a los supuestos enfermos. Además, disponen con los consultorios médicos de una red de distribución muy extendida y extremadamente eficaz para difundir sus productos entre el público y constituirse una clientela fiel. Por otra parte, sin embargo, el control que tiene la medicina sobre sus productos puede constituir para las farmacéuticas una limitación. Para vender drogas recreativas o moléculas de confort (lifestyle drugs), como el Viagra ${ }^{\circledR}$, la industria tiene interés en dirigirse directamente al consumidor, sin pasar por la mediación de la medicina.
Pero, ¿es realmente necesaria la categoría de enfermedad? Germund Hesslow (1993) afirma que no. De ser así, si abandonáramos la distinción entre salud y enfermedad, los laboratorios no podrían ni necesitarían vender enfermedades para vender sus fármacos. Se evitaría de ese modo la manipulación y la instrumentalización de las categorías y representaciones de la salud en nombre de intereses económicos, políticos y sociales, aunque probablemente aparecerían nuevos problemas. La medicina también perdería gran parte de su poder de control social. En efecto, la competencia exclusiva que tiene para decidir sobre temas de salud y enfermedad, dibujando fronteras y estableciendo clasificaciones, constituye para la medicina una fuente de poder fabulosa. La vida cotidiana de millones de personas depende de ese tipo de decisiones.

\section{CONCLUSIONES}

Hemos comprobado en la primera parte que existen muchas maneras de manipular la frontera entre la salud y la enfermedad. Nos hemos centrado en el problema del disease mongering porque presenta ejemplos claros de ficciones patrocinadas por la industria. Pero quienes han creado esas ficciones para las farmacéuticas, esto es, la comunidad médica internacional (a través de algunos de sus miembros), son quienes a la vez detienen la máxima autoridad para decidir, en materia de salud, qué enfermedades son reales y cuáles no. $Y$ esa autoridad les es concedida por la sociedad en su conjunto. Por consiguiente, desde una postura constructivista, deberíamos admitir que las llamadas "enfermedades ficticias" son a la vez enfermedades reales en la medida en que han sido reconocidas como tales por quienes las han creado.

En la segunda parte, hemos visto que la producción de "ficciones" médicas obedece a mecanismos más complejos, algunos de ellos inconscientes e involuntarios. Por un lado, el hecho de que existan moléculas eficaces para tratar algunas dolencias, así como el propio proceso de la investigación clínica, influyen en la categorización de aquello que curan. Y en ese proceso intervienen tanto los médicos investigadores como los pacientes. Por otro lado, la existencia de una enfermedad, más allá de los hechos en los que eventualmente se sustenta, depende de un conjunto de representaciones en las que intervienen todos los actores. En el caso de los trastornos mentales, cuando no existe una base biológica, las representaciones lo son casi todo. Desde la postura constructivista fuerte, donde no hay realidad más allá de aquello que nos representamos como tal, deberíamos pues reconocer 
que esas enfermedades son plenamente reales. ¿Pero de qué realidad estamos hablando?

Un quark, una bacteria, un préstamo hipotecario y una enfermedad no son menos reales unos que otros, pero pertenecen a diferentes tipos de realidad. La enfermedad tiene a menudo un componente biológico, y por lo tanto físico, pero también un componente so- cial y cultural, así como un componente psicológico que es la experiencia subjetiva propia de cada uno. La contribución respectiva de lo biológico y lo cultural en la experiencia subjetiva será más o menos grande en función del individuo y en función del contexto, pero esa experiencia no se reduce estrictamente a sus condicionantes objetivos, cuando los hay.

\section{NOTAS}

1 En este artículo usaremos las dicotomías "salud/enfermedad" y "normal/ patológico" como si fueran equivalentes aunque un examen más detallado mostraría que no lo son. Ambas son problemáticas, pero no del mismo modo. Dicho esto, el matiz en cuestión no es relevante para nuestro propósito.

2 La homosexualidad dejó de ser considerada como enfermedad mental por la Organización Mundial de la Salud con la publicación en 1992 de su décima revisión de la Clasificación Internacional de Enfermedades (ICD-10). Esta revisión fue aprobada el 17 de mayo de 1990 y entró en vigor para los países miembros en 1994. Anteriormente, en 1973, la American Psychiatric Association (APA) había retirado la homosexualidad de la lista de los trastornos, en la sección de desviaciones sexuales, del Diagnostic and Statistical Manual of Mental Disorders (DSM-II). Esa modificación se hizo supuestamente bajo la presión de los colectivos de homosexuales.
3 Ivan Illich criticaba en su Némésis Médicale (1975) la medicalización de la sociedad y de la vida misma. La película THX 1138 de George Lucas muestra que en 1971 ya existía una preocupación por el control farmacológico de la existencia. Ese tema también está presente en la novela Un mundo feliz (A Brave New World), de Aldous Huxley, escrita en 1932. El negocio de la enfermedad es ilustrado en 1923 por Knock, el médico de la novela de Jules Romains, y ejemplos todavía anteriores pueden encontrarse. Ahora bien, la primera autora en analizar el fenómeno actual del disease mongering, es decir la promoción de enfermedades con fines de lucro, parece ser Lynn Payer (1992). Desde entonces, tal vez como reacción ante la multiplicación de los escándalos farmacológicos, la publicación de libros y artículos se ha disparado. Desde 2002, la revista British Medical Journal es uno de los espacios de expresión más activos sobre ese tema. Y en 2006 fue organizado un primer congreso internacional.

4 Los efectos secundarios de los medicamentos, dice el comité, son responsa- bles del $5 \%$ de todos los ingresos en los hospitales del Reino Unido.

5 En 1987, el Manual diagnóstico y Estadístico de los trastornos mentales (DSM-III-R) recogía una única categoría de Trastorno Autista. En 2000, la edición siguiente del Manual (DSM-IV-TR) definía cinco categorías diagnósticas dentro de los Trastornos Generalizados del Desarrollo. En 2013, la última edición (DSM-5) vuelve a definir una sola categoría, el Trastorno del Espectro del Autismo, con criterios de diagnóstico modificados. Esta última revisión responde en parte a inconsistencias de diagnóstico.

6 Dicho sea de paso, en su cuarta edición, el $56 \%$ de los autores de ese manual tenían vínculos económicos con la industria farmacéutica; el $100 \%$ en el caso de los grupos de trabajo sobre "trastornos del humor" (mood disorders) y sobre "Esquizofrenia y otros trastornos psicóticos" (Cosgrove, Krimsky, Vijayaraghavan \& Schneider, 2006). 
Angell, M. (2005). The Truth about the Drug Compagnies: how they deceive us and what to do about it. Is the Party Over? New York: Random House Trade Paperbacks.

Boorse, C. (1975). "On the distinction between disease and illness". Philosophy \& Public Affairs, 5, 49-68.

Canguilhem, G. (1966). Le normal et le pathologique. Paris: PUF.

Cassels, A. \& Moynihan, R. (2005). Selling Sickness. How Drug Companies Are Turning Us All Into Patients. Crows Nest: Allen \& Unwin.

Coe, J. (2003). Healthcare: The lifestyle drugs outlook to 2008, unlocking new value in wellbeing. London: Reuters Business Insight.

Cosgrove, L.; Krimsky, S.; Vijayaraghavan, M. \& Schneider, L. (2006). "Financial ties between DSM-IV panel members and the pharmaceutical industry". Psychotherapy and Psychosomatics, 75 (3), 154-160

Delate, T.; Simmons, V. A. \& Motheral, B. R. (2004). "Patterns of use of sildenafil among commercially insured adults in the United States": 1998-2002. Int J Impot Res, 16 (4), 313-318.

Engelhardt, H. T. (1975). "The concepts of health and disease". En Evaluation and explanation in the biomedical sciences (pp. 125-141). Springer. Recuperado a partir de http://link.springer.com/chapter/10.1007/978-94-010-1769-5_9

Ereshefsky, M. (2009). “Defining 'health'and 'disease'". Studies in History and Philosophy of Science Part C, 40 (3), 221-227.
Forcades i Vila, T. (2006). Los crímenes de las grandes compañías farmacéuticas. Barcelona: Cristianisme i Justícia.

Foucault, M. (1961). Histoire de la folie à l'âge classique. Paris: Gallimard.

Hacking, lan. (2001). ¿La construcción social de qué? Barcelona: Paidós.

Hacking, Ian. (2004). "La fabrication des malades". La Recherche, (Hors-série 16), 46-48.

Hesslow, G. (1993). "Do we need a concept of disease?". Theoretical medicine, 14 (1), 1-14.

Hoffman, B. (2001). "Complexity of the Concept of Disease as Shown through Rival Theoretical Frameworks". Theoretical Medicine and Bioethics, 22, 211 236.

House of Commons Health Committee. (2005). The Influence of the Pharmaceutical Industry. London: House of Commons.

Illich, I. (1975). Némésis médicale: l'expropriation de la santé. (J.-P. Dupuy, Trad.). Paris: Seuil.

Laumann, E. O.; Paik, A. \& Rosen, R. C. (1999). "Sexual dysfunction in the United States: Prevalence and predictors". JAMA: Journal of the American Medical Association, 281 (6):537-44.

Lexchin, J. (2006). "Bigger and Better: How Pfizer Redefined Erectile Dysfunction". PLoS Medicine, 3 (4), e132.

Moynihan, R. (2003). "The making of a disease: female sexual dysfunction". British Medical Journal, 326, 45-47.
Moynihan, R. \& Henry, D. (2006). “The Fight against Disease Mongering: Generating Knowledge for Action". PLoS Medicine, 3 (4), e191.

Murphy, D. (2009, junio). "Concepts of Disease and Health". En E. N. Zalta (ed.), The Stanford Encyclopedia of Philosophy. Recuperado a partir de http:// plato.stanford.edu/archives/sum2009/ entries/health-disease/

Payer, L. (1992). Disease-Mongers: How Doctors, Drug Companies, and Insurers Are Making You Feel Sick. New York: WiIley and Sons.

Pignarre, P. (2001). Comment la dépression est devenue une épidémie? Paris: La Découverte.

Royal College of General Practitioners. (2004, agosto 17). RCGP warns against unhealthy pharmaceutical industry. Recuperado 13 de julio de 2011, a partir de http://www.rcgp.org.uk/default. aspx?page $=1609$

Sen, A. (2002). "Health: perception versus observation". British Medical Journal, 324 (7342), 860

Tiefer, L. (2006). "Female Sexual Dysfunction: A Case Study of Disease Mongering and Activist Resistance". PLOS Medicine, 3 (4), e178.

Wakefield, J. C. (1992). The concept of mental disorder: on the boundary between biological facts and social values. American Psychologist, 47 (3), 373. 